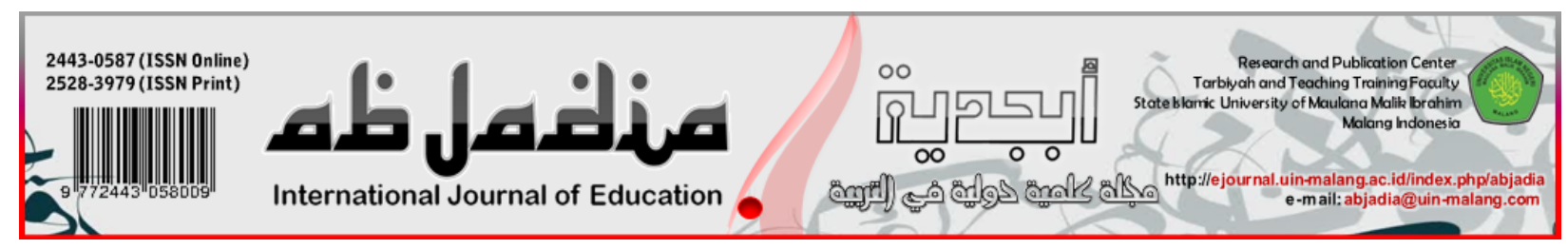

\title{
USING THE PICTURE AND PICTURE LEARNING STRATEGY IN LEARNING WRITING SKILL AT ISLAMIC STATE JUNIOR HIGH SCHOOL 5 OF BOJONEGORO
}

\section{استخدام استراتيجية التعلم الصورة والصهورة في تعليم مهارة الكتابة بالمدرسة المتوسطة الإسلامية الحكومية الخامسة بوجونغارا}

\author{
Sri Wahyuni ${ }^{*}$, Dewi Chamidah², Bisri Mustofa ${ }^{3}$ \\ Universitas Islam Negeri Maulana Malik Ibrahim Malang, Indonesia
}

Article History:

Received : 2021-10-04

Revised : 2021-12-14

Accepted : 2021-12-20

Published : 2021-12-26

\section{Keywords:}

Learning Strategy Picture and Picture, Writing Skill

\section{*Correspondence Address:} yuniealfi@gmail.com

\begin{abstract}
Picture and Picture is one of the learning strategies that use picture media in education. This strategy is active, creative and fun. Writing skills is one of the important in Learning Arabic. However the students of seventh class Islamic state Junior High School 5 Bojonegoro have many difficulties in writing sentences or simple paragraphs, because they feel bored and learning strategies used low attractive. The input in this research is quantitative and the research method used is the experimental method. The objectives of the research are to find out the use of picture and picture learning strategy in teaching writing skill in MTsN 5 Bojonegoro and to calculate the measurement of effectiveness of using picture and picture learning strategy in developing writing skill in MTsN 5 Bojonegoro. The input in this research is quantitative and the research method used is the experimental method. The results of this research are that the use of the picture and picture strategy is "effective", considering the result of the castration $\mathrm{t}$ : 11.75 greater than the degree of $\mathrm{t}$ tables at the $1 \%$ level $(2,000)$, and greater than the degree of $t$-tables at the $5 \%$ level $(2,660)$. By that, $(\mathrm{Ha})$ are acceptable, and $(\mathrm{H} 0)$ is rejected, meaning that the use of the Picture and Picture strategy is "effective".
\end{abstract}

\section{مقلم مقلة}

اللغة العربيّة هي إحدى اللغات المولودة من اللغة السامية حتى تتطّور من وقت إلى أخر. عند دكتور

محمد عبد العزيز"ان اللغة العربيّة ليست لغة حديثة هي لغة قديمة ولغة القرآن، وحيث إن القرآن أنزل

للنّاس كافة فلغتاه وجب أن تعرف للنّاس كافة، حيث إن العربيّة هي أصل جميع اللغات. و واللغة العربية لها أربع مهارات، وهي مهارة الاستماع ومهارة الكلام ومهارة القراءة ومهارة الكتابة. الكتابة احدي المهارات الأسـاسية في تعليم اللغة الأولية واللغة الأجنبية علي حد سواء. وكانت مهارة الكتابة هي المهارة والمحصلة النهائية من مهارات اللغوية الأربع، وتطبيق المهارات الأخرى. مهارة الكتابة هي عنصر من عناصر اللغة العبية الهامة في اللغة العربية ـ إما من ناحية الأصوات أو المفردات أو الصرف أو النحو. وفي هذا المبحث من حيث 
المفردات. الكتابة وسيلة الاتصال اللغوي بين الأفراد في التعبير عما لديهم من معان ومفاهيم ومشاعر وتسجيل الحوادث والوقائع. وكثيرا ما كان الخطأ في الرسم الكتابي أو في عرض الفكرة، سببا في تغيير المعنى وعدم وضوح الفكرة .

المدرسـة المتوسطة الإسـلامية الحكومية الخامسة ببوجونغارا هي إحدى المدارس التي تقدم دروسًا في

اللغة العربية لطلابها بدءًا من الصف السابع إلى الصف التاسع. تعلم التلاميذ فهها اللغة العربية ثلاث حصص في الأسبوع. كثير من التلاميذ في فصل السابع تخرجوا في المدارس الابتدائية الذين لا يتعلمون على اللغة العبية. فاعتبر التلاميذ الكتابة في اللغة العبية صعبة لذلك شعروا بالملل و السأم عندما اشتركوا في عملية تعليم اللغة العربية. خاصية في الصف السابع الذين واجهوا صعوبة كبيرة في تدريس مهارة الكتابة كترتيب الكلمات والجملة، وترتيب الجمل في فقرة أو أقسام بسيطة .

فمن المشكلات السابقة احتاج المعلم إلى الوسائل المعينة، والمداخل والاستراتيجية في تدريسها. الصيورة والصورة هو من احدي استراتيجية التعلم التي تستخدم الوسائل الصهورة في التعليم، اختصت هذه الاستراتيجية بنشط وإبداعي ورائع. تطبيق التعلم باستخدام الصورة والصورة فعالية لترقية كفاءة

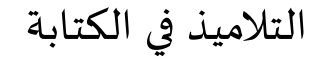

نظرا من مشكلة البحث السابقة تقدمت الباحثة أسئلة البحث كما يلي: (1) كيف استخدام استراتيجية التعلم الصورة والصورة في تعليم مهارة الكتابة بالمدرسة المتوسطة الإسلامية الحكومية الخامسـة ببوجونغارا؟ (2) ما مدي فعالية استخدام استراتيجية التعلم الصورة والصورة في تنمية مهارة الكتابة بالمدرسة المتوسطة الإسلامية الحكومية الخامسة ببوجونغارا؟

\section{[ن]}

استخدمت الباحثة المدخل الكمي بنوع التجريبي. المنهج التجريبي هو منهج البحث المستخدم لبحث

عن تأثير علاجات معينة على الآخرين. عند سوترسنو هادي، أن بحث التجريبي هو تصميم المجموعات العشوائية بالاختبار القبلي والاختبار البعدي. تصيميم البحث المستخدم في هذه البحث هو تصميم شبه تجريبي. يحتوي هذا التصميم على مجموعة ضابطة، لكناه ما عمل بكامل للتحكم في المتغيرات الخارجية التي تؤثر على تنفيذ التجريبية. في التصهيم شبه التجريبي ، شكل التصهيم شبه التجريبي المستخدم في هذا البحث هو تصميم المجموعة الضابطة غير المتكافئة. استخدمت الباحثة في هذا البحث مجموعتين 
هما المجموعة التجريبية والمجموعة الضابطة. تم التعامل في الفصل التجريبي باستخدام استراتيجية التعلم مع الصورة والصيورة. وأما مجموعاة الضابطة ستعطي مادة التعليم باستراتيجية عادياة.

مجتمع البحث هو كل الأشخاص والأشياء المتعلقة مع موضع مشكلة البحث . والمجتمع في هذا البحث هو التلاميذ الفصل السابع من المدرسة المتوسطة الإسلامية الحكومية الخامسـة ببوجونغارا في عام الدراسي 2020\2021. وعينتا التلاميذ في الفصل السابع "ه" وهم 34 تلميذا كالمجموعة الضابطة و34 تلميذا في الفصل السابع "و" كالمجموعاة التجريبية. واستخدام الباحثة تقنية أخذ العينات العشوائية. تنقسم متغيرات البحث قسمين، المتغير المستقل وسمى أيضا بالمتغير التجريبي هو استراتيجية التعلم الصيورة والصورة .والمتغير التابع في هذا البحث مهارة الكتابة. مصيادر في هذا البحث هي معلم اللغة العبية، أخذت الباحثة المعلومات بالملاحظة والمقابلة عن علمية التعليم والتعلم الذي شمل المدرس اللغة على الوسائل المستخدمة واستراتيجية تعليم مهارة الكتابة ومشكلاتها باستخدام استراتيجية الصورة والصهورة. والتلاميذ في بالمدرسة المتوسطة الإسلامية الحكومية الخامسـة ببوجونغارا. لجمع البيانات من التلاميذ لهذه المدرسـة بالمقابلة والملاحظة والاختبار والاستبانة عند التعليم والتعلم في ترقية مهارة الكتابة باستراتيجية الصورة والصورة. أما أسلوب جمع البيانات التي قامت بها الباحثة منها، 1) الملاحظة، 2) المقابلة، 3)الاستبانة هي الأسئلة المكتوبة للحصول على معلومات من عدد المستجيبين . ، 4) الاختبار، هو الأسئلة أو التمارين المستخدمة لقياس المهارات، ومعرفة الذكاء، والملكة لأفراد أو مجموعة .

لتحليل البيانات في آخر عملها استعملت التحليل الكمي. هذا التجليل لمعرفة فعالية استخدام استراتيجية التعلم الصورة والصورة في تعليم مهارة الكتابة في الفصل السابع بالمدرسة المتوسطة الإسلامية الحكومية الخامسـة ببوجونغارا. أما الرموز المستخدم هو الرموز المئوية والاختبار التائي (t-test). $t=\frac{M_{X}-M_{y}}{\sqrt{\left(\frac{\sum_{X} 2+\sum_{Y} 2}{N_{X}+N_{Y}-2}\right)\left(\frac{1}{N_{X}}+\frac{1}{N_{Y}}\right)}}$

نتائج 氩

استخدام استراتيجية التعلم الصهورة والصهورة في تعليم مهارة الكتابة بالمدرسة المتوسطة الإسلامية الحكومية الخامسة ببوجونغارا 
خطوات استعمال استراتيجية الصيورة والصيورة مصورة نحو التالي: (1)المقدمة، وهي بإلقاء السلام والتحية ثم الافتتاح بالبسملة والإدراك بالترابط الذي وجاه وركز التلاميذ موضوع المادة. (2).عرض المادة، في هذه المرحلة قدمت الباحثة المادة والقت الباحثة أهداف التدريس وشرحت استراتيجية التي استعملتها الباحثة في التعلم بالصورة و الصورة. (3) قسمت التلاميذ إلي الفرق وكل الفرقة تتكون من ستة أو سبعة تلاميذ. (4) عرض الصيور، قدمت الباحثة الصور، واحدا فواحدا علي السبورة، ثم وزعت الباحثة قرطاس الصورة كل الفرقة، أمرت الباحثة كل الفرقة بترتيب الصورة الصحيحة واحدا فواحدا من كل فرقة أن يتقدم أمام الفصل لترتيب الصورة. (5).أمرت الباحثة كل الفرقة تشرح الصورة، وفي هذه المرحلة سألت الباحثة آراء التلاميذ عن تسلسل الصور ثم يبينه، واحدي الفرقة يتقدم أمام الفصل ليشرح عن الصيورة حسب آراههم. (6). يعطى الباحثة الاقتراحات وشرحت وتأكدت الباحثة عن الصورة، أعطت الباحثة الاقتراحات عن سير المناقشة، شرحت وتأكدت الباحثة عن الصيورة من الصيورة الأولي حتي الصورة الأخيرة، إلقاء الباحثة الوظيفة بكتابة الجمل مستعينا بالصور. ثم تعطي الباحثة النتيجة. (7).الخلاصة والاختتام، استخلصت عن المادة ثم إلقاء الإرشادات على التعلم بالجدّ والاجتهاد. اختتمت بالحمدلة. في تطبيق استراتيجياة الصورة والصورة استعدت وسائل الصورة والصورة يعنى في شكل الصيور وفقا بالموضوع الدرس الذي طبق في الفصل. وهذا ناسب بنظرية مفهوم الصهورة والصورة وفقًا مفتاح الهدى ، الصورة والصيورة هي استراتيجية تعليمية استخدم الصور كوسائط تعليمية. هذه الصور هي الأدوات الرئيسية في عملية التعلم. قبل عملية التعلم ، وجب على المعلم تحضير صورة لعرضها، على شكل بطاقة أو مخطط كبير .

اختارت الباحثة وسيلة الصيور باستراتيجية الصيورة والصورة في هذا البحث لسهل التلاميذ في تعبير الكلمة في شكل الصهورة. والتلاميذ فهم شعروا بسعادة وأمكن التلاميذ للاهتمام بالأشياء أو الأمور التي تعلق بالدرس. والصورة أمكنت مساعدة المعلمين في تحقيق أهداف التعليم. التلاميذ في المجموعاة التجربية أنهم شعروا شعرا سعيدا تعليم اللغة العربية حاصة في تعلم مهارة الكتابة الموجهة بوسيلة استراتيجية الصورة والصورة. هذه النتائج دلت بأن استراتيجية الصيورة والصيورة استراتيجية لها تأثرات إيجابية. أن استراتيجية الصيورة والصورة استراتيجية مؤثرة بناء من نتيجة الاستبانة عرف بأن 94\% تلميذا شعروا بسرور باستخدام هذه الاستراتيجية. و 91\% من التلاميذ شعرون بسهلة في كتابة الجملة. 98\% شعرون بأن هذه الاستراتيجية يساعدهم في فهم المفردات في كتابة الكلمات. 97\% شعرون بأن هذه الاستراتيجية يساعدهم في كتابة الجملة بسهولة. 91\% شعرون أن هذه الاستراتيجية 
يساعدهم علي التعبير فكرة الرئيسية في كتابة الجملة. و94\% شعرون أن هذه الاستراتيجية مثيرة من استراتيجية التعلم الآخر. 94\% شعرون أن نتيجة التعليم أحسن باستخدام هذه الاستراتيجية. استراتيجية التعلم الصورة و الصورة من استراتيجية التعلم الآخر. 82\% شعرون بأن استخدام استراتيجية التعلم الصيورة و الصورة فيها تأثيرا عميق. و97\% شعرون بأن هذه الاستراتيجية تفيدهم كثيرا في تعليم الكتابة . التلاميذ موافقون بأن استخدام استراتيجية التعلم الصورة و الصورة جيد جدا، وساعدتهم في تعليم الكتابة الموجهة. التلاميذ يختارون الأجوبة موافق: 1،57\%، موافق بشدة:1،35\%، مشكوك: 5، \%\%، غير موافق:3،0\%. بمعني أن أكثرهم علي مستوي موافقون باستخدام استراتيجية التعلم الصورة و الصيورة في تعليم مهارة الكتابة.

فعالية استخدام استراتيجية التعلم الصهورة والصورة في تعلم مهارة الكتابة بالمدرسة المتوسطة الإسلامية الحكومية الخامسة ببوجونغارا

أما نتائج الاختبار القبلي والاختبار البعدي في التطبيق استراتيجية الصورة والصورة في الفصل السابع من المدرسة المتوسطة الإسلامياة الحكومية الخامسة بوجونغارا، فنتائج الاختبار البعدي للمجموعة الضابطة 34 تلميذا متساويا في قيمة المعدلة. نتيجة الاختبار القبلي للمجموعاة الضابطة بقيمة: 2485 وبمعدلة 26،72. فالنتيجة الاختبار البعدي للمجموعة الضيابطة بقيمة:2460 وبمعدلة: 72،35. أو بمعني ليس هناك الارتفاع من النتيجة الاختبار القبلي إلي النتيجة الاختبار البعدي للمجموعة الضـابطة. أما نتيجة الاختبار القبلي والاختبار البعدي للمجموعة التجريبية 34 تلميذا فهناك الارتفاع. نتيجة الاختبار القبلي في المجموعة التجربة بقيمة: 2460 وبمعدلة: 32،35، وفي الاختبار البعدي بقيمة المجموع: 2960 وبمعدلة: 87،06. فهذا دل علي أن ترقية كفاءة التلاميذ في الفصل التجريبي من الاختبار القبلي إلي الاختبار البعدي يعني بقيمة الدرجاة: 14 (14، (87،06 - 72،35= 14، 14، من النتيجة الاختبار القبلي إلي النتيجة الاختبار البعدي.

t أن نتيجة الاختبار القبلي والاختبار البعدي لها فرق إيجابي. أما نتيجتهما بتحليل الإحصاء هي

الإحصائي = 795،11. ونتيجة t الإحصائي مقارنة بتعيين Ttable فوجد أن نتيجة = 2،660 في المستوى المعنوي 1\% و 000،2 في المستوى المعنوي 5\%.. اختبار t هو اختبار تم إجراؤه لاختبار الفرضيات في بحث علاجي واحد سونديانا( 2016).= (2000>795،11>2,660). فإذا نطرنا تقرير "ت:"

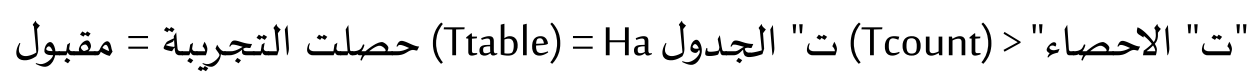




$$
\text { "ت" الاحصياء" > (Ttable) = HO فشلت التجريبة = مردود. }
$$

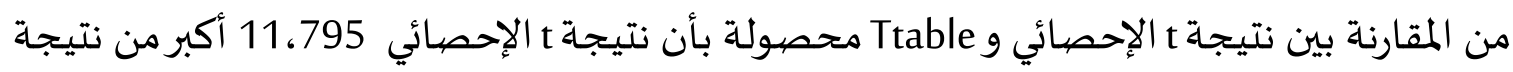
في المستوى المعنوي 1\% 660،2. وكذلك أكبر من نتيجة في المستوى المعنوي 5\% يعني 000،2،. والخلاصة هذه بمعنى أن HO مردود، و Ha مقبول يعنى أن فرضية هذا البحث "مقبول". عرفنا أن مضمون Ha استخدام استراتيجياة الصورة والصورة في تعليم مهارة الكتابة لدى التلاميذ في المدرسة المتوسطة الإسلامية الحكومية الخامسة ببوجونغارا فعّال. و Hoاستخدام استراتيجية الصورة والصورة في تعليم مهارة الكتابة لدى التلاميذ في المدرسـة المتوسطة الإسلامية الحكومية الخامسة ببوجونغارا غير فعّال. بناء على نتيجة كفاءة التلاميذ في مهارة الكتابية من الفصل التجريبي والفصل الضابط هناك اختلاف بين الفصل الذي يتعلم اللغة العربية باستخدام استراتيجية التعلم الصورة و الصورة والفصل الذي يتعلم اللغة العربية بغير استخدام استراتيجية التعلم الصورة والصورة، كوسيلة التعلم في ترقية كفاءة التلاميذ في كتابة الكلمات أو الجمل. حي تستطيع الباحثة أن تحلل فعالية استخدام استراتيجية التعلم الصورة و الصورة في ترقية كفاءة التلاميذ بالمدرسة المتوسطة الإسلامية الحكومية الخامسة بزجونغارا عام الدراسي 2020\2021 في كتابة العربية تأسيسا من نتيجة هذا البحث. وبناء على نتيجة الاستبانة في الفصل التجريبي دل علي أن التلاميذ أكثرهم موافقون بأن استخدام استراتيجية التعلم الصيورة و الصورة جيد جدا، لأن هذه الاستراتيجية ساعدهم في تعليم الكتابة وفعالية في نشاط التعلم وترقية كفاءة التلاميذ في مهارة الكتابة. وعرفنا من اللوحات السابقة أن التلاميذ اختاروا الأجوبة بموافق و موافق بشدة أكثر من الأجوبة غير موافق (موافق:1،57\%، موافق بشدة:1،35\%، مشكوك: 5، 7 ،غير موافق:3،0\%). فهذا بمعني أن أكثرهم علي مستوي موافقون باستخدام استراتيجية التعلم الصورة و الصورة في تعليم مهارة الكتابة.

فعالية استراتيجية الصورة والصورة في تعليم مهارة الكتابة بالمدرسة المتوسطة الإسلامية الحكومية الخامسة ببوجونغارا أن ننظر إلى نتيجة الاستبانة بأههم يشعرون السهل في مهارة الكتابة بوسيلة استراتيجية الصيورة والصيورة. يناسب بالنظرية أنّ استراتيجية التعليم هي المنهج الذي اختاره المدرس في عملية التعليم الذي تقدر أن تعطى السهولة أو اليسر للتلميذ حتى يتحقق هدف التعليم. وفي حين يفسر كيمب (1995) أن استراتيجيات التعلم نشـاط لتعلم أن تفعل المعلمين والتلاميذ حتى التعلم الأهداف يمكن تحقيق فعالية 
إن تطبيق استراتيجية الصورة والصهورة في تعليم مهارة الكتابة بالمدرسة المتوسطة الإسلامية الحكومية الخامسة بوجونغارا وفقا بخلفية التلاميذ ليسهل في تعبير الكلمة على شكل الصورة. والتلاميذ شعروا بالسعادة وتمكن التلاميذ للاهتمام بالأشياء أو الأمور التي تتعلق بالدرس. والصيورة مساعدة المعلمين في تحقيق أهداف التعليم. فينبغي أن يُطبق استراتيجية الصورة والصيورة في تعلم مهارة الكتابة مناسبا باحتياجات التلاميذ. استراتيجية التعلم الصورة والصورة فعالة لترقية كفاءة التلاميذ في تعليم مهارة الكتابة وهذه يسهل التلاميذ في كتابة ترتيب الجمل و كذلك كتابة إنشاء الموجهة. وأن استخدام استراتيجية التعلم الصورة والصورة في تعليم مهارة الكتابة بالمدرسة المتوسطة الإسلامية الحكومية الخامسة بوجونغارا فعالة. بالدليل أن نتيجة التاء الإحصائي أكبر من نتيجة التائي الجدول المئوي 1\% و 5\%.

\section{مراجع}

أحمد خيرى محمد كاظم و جابر عبد الحميد جابر، الوسيائل التعليمية و المنهج، القاهرة: دار

$$
\text { النهضية العربية، } 1997
$$

أحمد فؤاد عليان، المهارات اللغوية ماهيتها وطرائق تلسريسها. الرياض: دار المسلم المنشر والتوبع. 1992

بشير عبد الرحيم الكلوب، الونائل التعليمية التعليمية: إعلدادها وطرق استخدلمها، عمان: مكتبة المحتسبب، 1986

بطاقات ألعاب لغوية كوسيلة تعليمية ، مأخوذ في 23 مارس 2009 جابر عبد الحميد و الآخرون، منابج البحث في التربية و علم التفس، القاسرة: دار الدهضية العربية، 1978

حسن شحاته .تعليم اللغتة العبرية بين النظرية والتطبيقية. القاهرة: الدار المصرية اللبنانية. 2002

حسين حمدي الطوبجى، وسائل الاتصال و التكنولوجيا في التعليه، الكويت : دار القله، 1987 
حنيفة ، أمي ، فعالية تطبيق طريقة الصورة والصيورة في تعليم مهارة الكلام لطلاب الفصل السـابع بمدرسـة زين الدين المتوسطة سيدوارجو".كليات دراسـة العليا بجامعة

الإسلامية الحكومية سونان أمبل سـورابايا، 2014

رشـدي أحمد طعيمة، تعليم العبرية لغير الناطقين بها مناهجه وأساليبه. الرياط: منشورات المعظمة السلامية للتربية والعلوم والثقافة، 1989.

زكريا ابراهيم. دون السنة. طرق التسريس اللغة العربية. دار المعرفة الجامعة.

عبد الرحمن احمد البورزي، اللغة العربية أقل اللغات كلها، الطبعة الأولي، الأردن، دار الحسن للنشر والتوزع، 1998م

عبد العزيزبن زيد ابو تيلي، الوسائل التعليمية (حقيقية تدريبية)، الرياض: المراجعة العلمية

والفنية وحدة التطوير بإدارة التديب التربوي، 1420 هـ

علي أحمد مدكور، تدريس اللغة العربية ط. .1الكويت: مكتبة الفلاح، 1983 على أحمد مدكور، تدريس فنون اللغة العربية. القاهرة: دار الفكرى العربى، 2002. محمد علي الخولي، أسليب تلدريس اللغة العربية ، الرياض: المملكة العربية السعودية، 1982 محمد صالح الشنط، المهارة اللغوية مدخل الى خصيائص اللغة العببية وفنونها. حائل: دار الندلوس للنشر التوزيع. 1996.

محمود ابراهيم الخطيب، طرائق تعليم اللغتة العببية. رياض: مكتب التوبة، 2003.

Anshor, Ahmad Muhtadi.Pengajaran Bahasa Arab Media dan MetodeMetodenya.Yogyakarta: Sukses Offset.2009

Arikunto, Suharsimi.Prosedur Penelitian Suatu Pendekatan Praktik.Jakarta: Rineka Cipta. 2010

Fauzi, Rahmat, Dwiastuti, Sri dan Harlita. Penerapan Metode Picture and Picture untuk Meningkatkan Motivasi Belajar Biologi Siswa Kelas VII DSMP 14 Surakarta. Jurnal Pendidikan Biologi, Volume 3 no 3. 2011

Firdaus,Z.M.,Belajar Cepat dengan Diskusi, Metode Pengajaran efektif di Kelas, Surabaya: Indah, 2009.

Hamdani, Strategi Belajar Mengajar. Bandung: CV Pustaka Setia.2011.

Hamzah, B.Uno dan Nurdin Muhammad, Belajar dengan Pendekatan Paikem Jakarta:PT Bumi aksara, 2020.

Hanifah, Umi. " تطبيق طريقة الصورة والصورة)(Picture and Picture) في تعليم مهارة الكلام Prosiding Konferensi Nasional Bahasa Arab dan Pembelajarannya di Era Milenial (2019. 
Hakim, M. Arif Rahman, et al. "The Utilization Of Picture and Picture Strategy: An Effective Way to Improve EFL Students' Writing Ability in Madrasah Tsanawiyah." Madania: Jurnal Kajian Keislaman 24.1 (2020): 31-38

Hermawan, Acep.Metodologi Pembelajaran Bahasa Arab.Bandung: PT. Rosda Karya.2011

Huda, Miftahul. Model-Model Pengajaran dan Pembelajaran Isu-Isu Metodis dan Paragmatis, Yogyakarta: Pustaka Pelajar,2019

Isjoni.Pembelajaran Kooperatif,.Yogyakarta: Pustaka Pelajar.2009

Istarani.Model Pembelajaran Inovatif Referensifi Guru Dalam Menentukan Model Pembelajaran.Medan: Media Persada.2011

Izzan, Ahmad.Metodologi Pembelajaran Bahasa Arab.Bandung: Humaniora.2004

Kusumah, Wijaya dan Dwitagama, Dedi. Mengenal Penelitian Tindakan Kelas Edisi: 2. Jakarta: PT Indeks.2011

Munip, Abdul, Penilaian Pembelajaran Bahasa arab, Yogyakarta: Fakultas Tarbiyah UIN Sunan Kalijaga, 2017.

Mustofa,Bisri dan Hamid,M.Abdul, Metode Dan Strategi Pembelajaran Bahasa Arab, Malang: UIN Maliki Press, 2020

Mustofa, Syaiful.Strategi Pembelajaran Bahasa Arab Inovatif. Malang:UIN Maliki Press Malang dalam Qomaruddin,hmad.Implementasi Metode Menyanyi dalam PembelajaranMufrodat,Jurnal Kependidikan, Vol.5 No.1

Nurmiati, Andi. Peningkatan Kemampuan Menulis Karangan Eksposisi Dengan Menggunakan Strategi Picture and Picture Siswa Kelas VIII-6 Smp Negeri 12 Makassar." Jurnal Konfiks 1, 2014.

Robertus, Angkowo dan Kosasih, A. Optimalisasi Media Pembelajaran.Jakarta: Grasindo.2007

Rohani, Ahmad.Media Instruksional Edukatif.Jakarta: Rineka Cipta.1997

Sadiman, Arief dkk, Media Pendidikan.Jakarta: Grafindo Persada.2003

Sugiyono, Metode Penelitian Kuantitatif Kualitatif dan RED.Bandung: Alfabeta.2011

Suprijono, Agus.Cooperative Learning Teori dan Aplikasi Paikem. Jakarta: Rineka Cipta.2009

Suryani, Nunuk dan Agung, Leo. Strategi Belajar Mengajar. Yogyakarta: Penerbit Ombak Dua. 2012

Sulaiman, Amir Hamzah. Media Audio Visual Untuk Pengajaran, Penerangan dan Penyuluhan.Jakarta: Gramedia, 1988

Trianto. Model-Model Pembelajaran Inovatif Berorientasi Kontruktivistik Prestasi. Jakarta : Pustaka Belajar.2007 
Sri Wahyuni, Dewi Chamidah, Bisri Mustofa

Wahab, Abdul.Media Pembelajaran Bahasa Arab.Malang: UIN Press.2009

Wena, Made.Strategi Pembelajaran Inovatif Kontemporer.Jakarta : Bumi Aksara, 2011

Widyawati, Wiwik Yulli, "Keefektifan Pembelajaran Picture and Picture Dalam Keterampilan Menulis Untuk Tingkat Universitas", Jurnal Kredo Vol.2 No.2, (April, 2019).

Yusuf, A. Muri. Metode Penelitian Kuantitatif, Kualitatif \& Penelitian Gabungan. Jakarta: Kencana,2014.

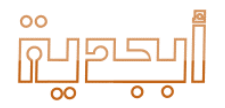

